\title{
Multiplex-GAM: genome-wide identification of chromatin contacts yields insights not captured by $\mathrm{Hi}-\mathrm{C}$
}

Robert A. Beagrie ${ }^{1,2, *}$, Christoph J. Thieme ${ }^{1, *}$, Carlo Annunziatella ${ }^{3, *}$, Catherine Baugher ${ }^{4}$, Yingnan Zhang, ${ }^{4}$ Markus Schueler ${ }^{1}$, Alexander Kukalev ${ }^{1}$, Rieke Kempfer ${ }^{1}$, Andrea M. Chiariello $^{3}$, Simona Bianco ${ }^{1,3}$, Yichao $\mathrm{Li}^{4}$, Antonio Scialdone ${ }^{5,6,7}$, Lonnie R. Welch ${ }^{4, \hbar}$, Mario Nicodemi ${ }^{1,3,8, \ddagger}$, Ana Pombo ${ }^{1,8,9, 末}$

Addresses:

${ }^{1}$ Epigenetic Regulation and Chromatin Architecture Group, Berlin Institute for Medical Systems Biology, Max-Delbrück Center for Molecular Medicine, Hannoversche Str. 28, 10115 Berlin, Germany.

${ }^{2}$ Laboratory of Gene Regulation, Weatherall Institute of Molecular Medicine, Oxford, OX39DU, UK.

${ }^{3}$ Dipartimento di Fisica, Università di Napoli Federico II, and INFN Napoli, CNR-SPIN, Complesso Universitario di Monte Sant'Angelo, 80126 Naples, Italy.

${ }^{4}$ School of Electrical Engineering and Computer Science, Ohio University, 45701 Athens, $\mathrm{OH}$, USA.

${ }^{5}$ Institute of Epigenetics and Stem Cells, Helmholtz Zentrum München - German Research Center for Environmental Health, Munich, 81377, Germany

${ }^{6}$ Institute of Functional Epigenetics, Helmholtz Zentrum München - German Research Center for Environmental Health, Neuherberg, 85764, Germany

${ }^{7}$ Institute of Computational Biology, Helmholtz Zentrum München - German Research Center for Environmental Health, Neuherberg, 85764, Germany

${ }^{8}$ Berlin Institute of Health (BIH), MDC-Berlin, Berlin, Germany.

${ }^{9}$ Humboldt-Universität zu Berlin, 10117 Berlin, Germany.

*Joint first authors, ${ }^{\ddagger}$ Joint corresponding authors. 


\section{Summary (Abstract)}

Technologies for measuring 3D genome topology are increasingly important for studying mechanisms of gene regulation, for genome assembly and for mapping of genome rearrangements. Hi-C and other ligation-based methods have become routine but have specific biases. Here, we develop multiplex-GAM, a faster and more affordable version of Genome Architecture Mapping (GAM), a ligation-free technique to map chromatin contacts genome-wide. We perform a detailed comparison of contacts obtained by multiplex-GAM and Hi-C using mouse embryonic stem ( $\mathrm{mES}$ ) cells. We find that both methods detect similar topologically associating domains (TADs). However, when examining the strongest contacts detected by either method, we find that only one third of these are shared. The strongest contacts specifically found in GAM often involve "active" regions, including many transcribed genes and super-enhancers, whereas in Hi-C they more often contain "inactive" regions. Our work shows that active genomic regions are involved in extensive complex contacts that currently go under-estimated in genome-wide ligation-based approaches, and highlights the need for orthogonal advances in genome-wide contact mapping technologies.

\section{Introduction}

Our understanding of gene regulation has been dramatically transformed by genome-wide technologies for identifying regulatory elements (e.g. ChIP-seq, ATAC-seq; Andersson and Sandelin, 2020) and by technologies that reveal how these elements are connected to one another through 3D genome conformation (e.g. Hi-C; Kempfer and Pombo, 2020). However, many cell types of interest are too rare to assay using these methods. Although single-cell variants of Hi-C are available, they require purified, disaggregated cell suspensions which can be unachievable for rare cell types embedded within complex tissues. Furthermore, methods based on chromatin conformation capture (3C) usually focus on contacts between pairs of elements, neglecting higher-order associations. We previously showed that Genome Architecture Mapping (GAM) can identify three-way chromatin contacts and achieves strong enrichment for contacts between regions containing active genes, enhancers and superenhancers whilst requiring only a few hundred cells (Beagrie et al., 2017).

The original GAM protocol involves DNA sequencing of many individual thin nuclear slices (which we call nuclear profiles, or NPs), each isolated in a random orientation from a different cell in the population. The principle behind GAM is that DNA loci which are physically close to each other in the nuclear space are present in the same NP more frequently than loci which are remote from one another. In the prototype version of GAM, a collection of 200-nm cryosections were cut through a sample of mES cells, before microdissection of single NPs into separate PCR tubes, followed by lengthy manual preparation of sequencing libraries to determine the DNA content of each tube. 
We have made several significant improvements to GAM. First, to reduce the hands-on time required for sequencing hundreds or thousands of NPs, we developed multiplex-GAM. In this variant of GAM, multiple NPs can be added into a single tube and then sequenced together, cutting down on both labour and reagent costs. Second, we optimized the protocol for DNA extraction from NPs such that it is now compatible with liquid dispensing robots, further reducing time and reagent volumes required to generate a GAM dataset. Third, we extended the SLICE statistical model for analysis of GAM data to cover a wider range of experimental parameters, including the addition of multiple NPs per tube. We also use the SLICE statistical model to determine optimal experimental parameters to aid the design of GAM experiments in different cells and organisms. Fourth, we produce an extended GAM dataset from mES cells, which we use for comparisons with Hi-C. Finally, we show that many contacts are equally detected by both methods, but also identify method-specific contacts, especially those that involve simultaneous associations between three or more genomic elements. We show that GAM is a versatile methodology for mapping chromatin contacts, and provide a framework to design GAM experiments that considers the depth of chromatin contact information required and minimises data collection effort.

\section{Results}

We previously published a GAM dataset of 408 NPs from mES cells, each isolated from a different nucleus into a single PCR tube (Fig. 1a, original-GAM). We showed that the number of times particular genomic loci are found together in the same NP (their cosegregation) is a measure of their physical proximity in the original population of cells, with high co-segregation values indicating that the regions were close in space. In the first GAM dataset, loci on different chromosomes (which we assume will be distant in the nuclear space) are found together in less than $1 \%$ of NPs (Beagrie et al., 2017). We therefore reasoned that combining more than one NP into a single sequencing library would not reduce our ability to distinguish interacting from non-interacting loci (Fig. 1a, multiplex-GAM).

To test this intuition, we used a dataset of 481 NPs sequenced individually, which include 408 previously published samples (Beagrie et al., 2017) plus 73 additional NPs (Supplementary Table 1). To simulate multiplex sequencing of two or three NPs, we combined the 481 datasets from single NPs, and generated 240 or 160 in silico GAM samples containing two or three NPs, respectively. We then re-calculated co-segregation matrices from these simulated multiplex-GAM datasets and found that these were visually highly similar (Fig. 1b; Supplementary Fig. S1c).

To formally understand the effect of including several NPs in multiplex-GAM experimental designs, and to optimize the experimental parameters, we next updated SLICE, the statistical tool previously developed to infer non-random DNA interaction probabilities from locus cosegregation in GAM data (Beagrie et al. 2017). We considered the effects of number of NPs 
per GAM sample, nuclear ellipticity, and NP thickness (Fig. 1c, Supplementary Fig. 1a). To determine the optimal parameters for collection of multiplex GAM datasets in mES cells, we expanded the mathematical model SLICE (Beagrie et al., 2017), and estimated the minimal number of GAM samples $\left(m^{*}\right)$ required to detect chromatin contacts with a probability of interaction above a specific threshold $(P i)$, given the number of NPs per GAM sample $\left(X_{N P}\right)$, nucleus ellipticity, section thickness $(h)$ and genomic resolution (Fig. 1d, Supplementary Note). We find that $m^{*}$ increases for higher resolutions and for weaker interaction strengths (lower $P i$ ), as expected (Fig. 1e,f). We also find that $m^{*}$ remains constant over the range of cryosection thicknesses convenient for ultracryomicrotomy, while $X_{N P}$ decreases with increasing thickness (Fig. 1g). Finally, we show that the expected co-segregation frequency of two interacting loci is relatively invariant for all but the most extremely ellipsoidal nuclei (Supplementary Fig. 1b).

Using the updated SLICE model, we calculate optimal experimental parameters for the application of GAM to a range of different organisms and cell types. Despite differences in ploidy and nuclear geometry between the selected cell types, we find that the minimum number of tubes $\left(m^{*}\right)$ required to reach a given statistical power is approximately constant ( $\sim 150$ tubes to detect interactions with $P i \geq 30 \%$ ) provided that each sample is collected with the optimal number of multiplexed NPs (Supplementary Fig. 1c).

Finally, we determined the optimal experimental parameters for producing a new multiplexGAM dataset in mES cells, and found that $m^{*}$ reaches a minimum with a multiplexing factor of 3-10 NPs per GAM library (Fig. 1h). A GAM dataset of $\sim 250$ libraries each multiplexed with 3 NPs (i.e. obtained from a total of only $750 \mathrm{mES}$ cells) would be sufficient to sample contacts with interaction probabilities above $50 \%$ at resolution of $30 \mathrm{~kb}$ across genomic distances $>100 \mathrm{~kb}$, while reducing reagent costs by two thirds (Fig. 1h).

Next, we implemented an improved experimental pipeline for GAM data collection which incorporates staining of the cells for better identification, and shorter or improved steps to facilitate high-throughput experiments, such as storage of laser microdissected NPs, automated pipetting, DNA purification, amplification and sequencing (Fig. 1i). One challenge in the prototype version of GAM was that some cryosections intersect cells without intersecting the nucleus and therefore contain no genomic DNA. In our efforts to identify chemical stains that could allow direct visualisation of nucleus prior to microdissecion, we found that most DNA stains prevent subsequent extraction and/or amplification of DNA from NPs, probably because they bind too strongly or damage DNA (Supplementary Fig. 2a). We therefore collected most of the new multiplex-GAM dataset using a cresyl violet stain which greatly improves identification of cellular profiles during microdissection without affecting DNA extraction (but which does not distinguish the cytoplasm from the nucleus; Fig. 1j, Supplementary Fig. 2b).

In the absence of a direct nuclear stain, we next optimized our multiplex-GAM collection by determining the proportion of cellular slices devoid of NPs. We stained mES cell cryosections for DNA and RNA and found that $23 \%$ of profiles intersect the cytoplasm only, 
with no detectable nuclear material (Supplementary Fig. 2c). As our current GAM pipeline does not distinguish these "cytoplasmic profiles" from NPs, we calculated that if we dissected four cresyl violet stained profiles into each sequencing tube, on average three of those four profiles would intersect the nucleus (Supplementary Fig. 2d).
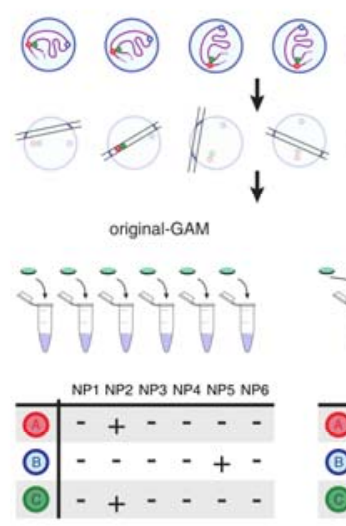

e

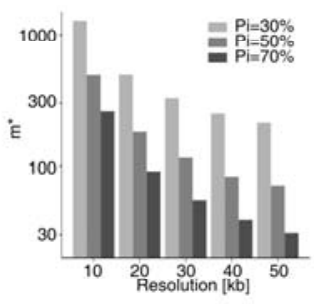

i

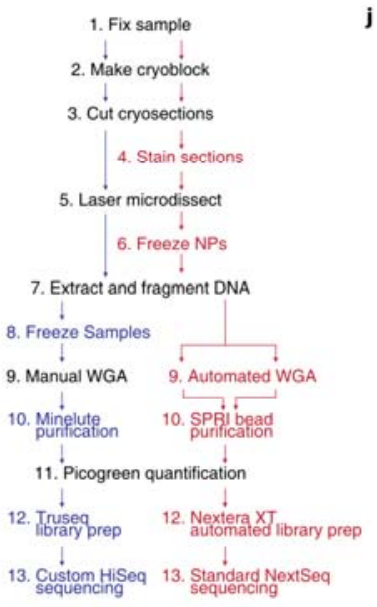

b

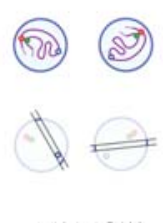

multiplex-GAM
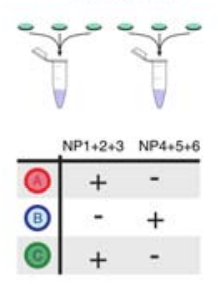

f

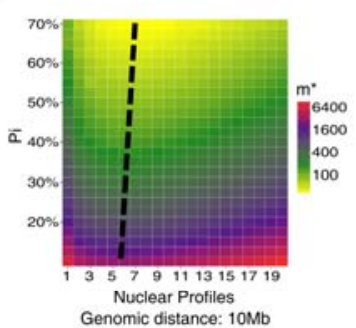

c Expanded SLICE modelling parameters

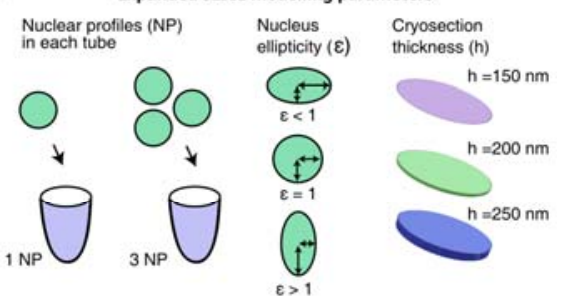

SLICE-informed optimization of GAM experiment design

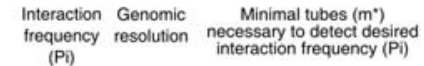
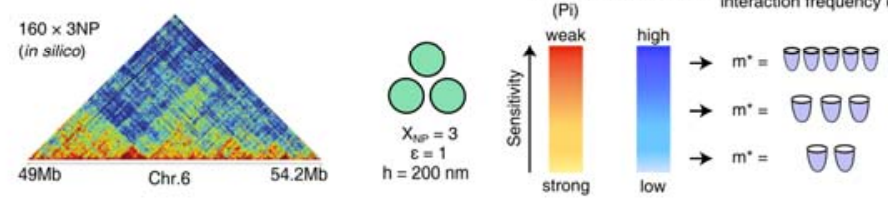

h

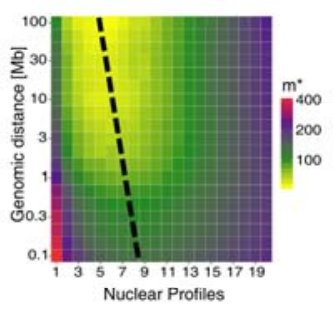

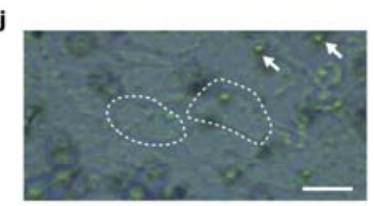

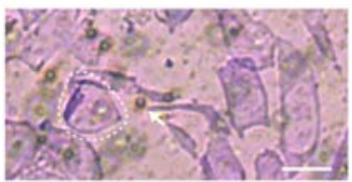

Figure 1

a, In a standard GAM experiment, thin slices from individual nuclei (nuclear profiles or NPs) are isolated by cryosectioning and laser microdissection, then the DNA content of each slice is determined by next generation sequencing. In a multiplex-GAM experiment, DNA from multiple NPs is extracted and sequenced together, reducing sequencing costs. b, Multiplex-GAM data constructed in silico by combining $1 \mathrm{NP}$ datasets at $40 \mathrm{~kb}$ resolution. c, SLICE accounts for the number of NPs multiplexed in each tube $\left(X_{N P}\right)$, the nuclear ellipticity (Supplementary Fig. S1a) and the thickness of each NP $(h)$ to estimate the minimal number of samples/tubes $\left(m^{*}\right)$ needed to achieve a given 
statistical power. d, Relationship between the number of tubes required $(m *)$, genomic resolution and sensitivity. e, Required number of tubes $\left(m^{*}\right)$ as a function of genomic resolution and interaction frequency $P i$ given a genomic distance of $10 \mathrm{Mb}$, three NPs per tube $\left(X_{N P}=3\right)$ and detection efficiencies estimated from the previously published GAM dataset at the corresponding resolution (Beagrie et al., 2017). f, $m^{*}$ as a function of $X_{N P}$ and the interaction probability ( $P i$ ) given perfect detection efficiency, a genomic distance of $10 \mathrm{Mb}$ and $h=220 \mathrm{~nm}$, at $30 \mathrm{~kb}$ resolution. Dashed black line marks the approximate position of the minimum value of $m^{*}$ across each row. $\mathbf{g}$, Optimal value of $X_{N P}$ and the corresponding value of $m^{*}$ for a range of possible slice thicknesses given $P i=30 \%$, genomic distance of $10 \mathrm{Mb}$ and perfect detection. $\mathbf{h}, m^{*}$ as a function of $X_{N P}$ and the genomic distance given perfect detection, $P i=50 \%$ and $h=220 \mathrm{~nm}$, at $30 \mathrm{~kb}$ resolution. Grey line marks the position of the minimum value of $m^{*}$ across each row. $\mathbf{i}$, Comparison of the original (black and blue lettering) and multiplex (black and red lettering) GAM protocols. WGA: Whole Genome Amplification. j, Top: unstained cryosections from mES cells. Individual NPs are outlined by dashed white lines. White arrows indicate typical background (air bubbles) in the microdissection membrane. Bottom: Cresyl violet stains both cytoplasm and nucleoplasm in $\mathrm{mES}$ cell cryosections as visualized by brightfield microscopy, and therefore does not distinguish between cellular profiles that do or do not intersect the nucleus.

To directly test the multiplex-GAM approach with our revised experimental pipeline, we collected a new batch of 249 multiplex GAM sequencing libraries, each containing three nuclear profiles on average, from an independent biological replicate from mES cells (Fig. 2a,b). The distribution of quality metrics (most importantly the percentage of mapped reads) was comparable across different collection batches (Supplementary Fig. 3a). The genomic coverage of this dataset ( $18 \%$ of $40 \mathrm{~kb}$ windows are detected per NP on average) was consistent with the expected presence of three NPs per library on average (7\% for single NPs, $20 \%$ for 3NP in silico data; Supplementary Fig. 3b) (Beagrie et al., 2017). Comparison of normalized linkage matrices between the 249x3NP multiplex-GAM dataset and the 481x1NP original-GAM dataset indicated that local contact information is well preserved in multiplexGAM, and conserved between biological replicates (Fig. 2a,b). Erosion analysis of the 3NPGAM dataset at $40 \mathrm{~kb}$ shows that its correlation with the $1 \mathrm{NP}-\mathrm{GAM}$ dataset resolution begins to saturate at $200 \times 3 \mathrm{NPs}$, indicating that the $249 \times 3 \mathrm{NP}$ dataset is large enough to explore chromatin structure at this resolution (Supplementary Fig. 3c).

We next considered the possibility of merging the $1 \mathrm{NP}$ and $3 \mathrm{NP}$ datasets to increase our power for in-depth comparisons between GAM and Hi-C data. First, we used the 1NP-GAM data to test in silico the effect of combining 1NP and 3NP data, and found that matrices from combined 1NP and 3NP libraries retain the quality of 3NP libraries (Supplementary Fig. 4a). Therefore, we merged the experimental $1 \mathrm{NP}$ and $3 \mathrm{NP}$ datasets to create a combined GAM dataset spanning a total of $1250 \mathrm{NPs}$, each from a different cell (Fig. 2c). To confirm the increased statistical power of the combined $1+3 \mathrm{NP}$ dataset, we used SLICE to determine the prominent interactions and compared them with those obtained with the original $408 \times 1 \mathrm{NP}$ dataset (Beagrie et al., 2017). Using the deeper $1250 \mathrm{NP}$ dataset from combined $1+3 \mathrm{NP}$ GAM data, we detect a greater number of prominent interactions compared to the published data (Fig. 2d,e). These prominent interactions showed a similar pattern of enrichment for genomic features as previously shown in our original 408x1NP mES cell dataset containing 
only 1NP samples, both for pairwise (Fig. 2f; Supplementary Fig. 4b) and three-way interactions (Fig. 2g).

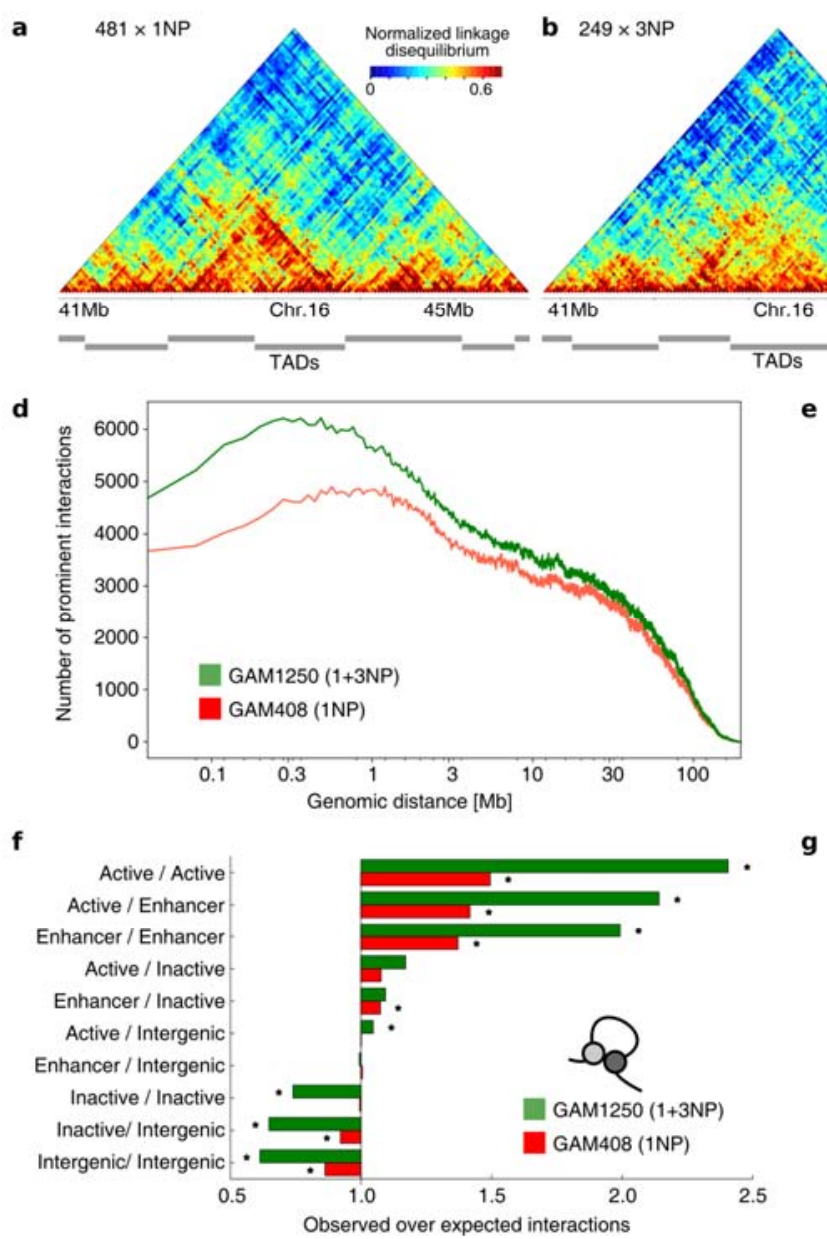

\section{Figure 2}

GAM matrices of normalized linkage with a resolution of $40 \mathrm{~kb}$ for a, multiplex-GAM using 3NPs (249 datasets), b, GAM using 1NPs (481 datasets), and c, the combined dataset of 1NPs and 3NPs. d, Number of prominent interactions identified by SLICE at each genomic distance in the merged GAM1250 dataset and the published mES GAM dataset (Beagrie et al., 2017). e, Contact matrix centered at the Sox 2 locus presenting prominent interactions identified by SLICE at $40 \mathrm{~kb}$ resolution. Functional elements in the locus are identified below the matrix, and previously identified interactions between these elements are identified by black arrows (Bonev et al., 2017). f, Enrichment analysis of pairwise interactions identified by SLICE involving active, inactive, intergenic or enhancer regions for the merged GAM-1250 dataset (green) and the original GAM-408 dataset (red). Statistically significant enrichments/depletions (those falling outside 95\% of randomised observations after Bonferroni correction) are marked by an asterisk. g, Enrichment analysis of triplet interactions identified by SLICE for the merged GAM-1250 dataset (green) and the original GAM-408 dataset (red).

One of the key aims of genome-wide 3D chromatin folding assays is the detection of TADs (Beagan and Phillips-Cremins, 2020). We used the insulation score method (Crane et al., 2015) to identify TAD boundaries in GAM and published Hi-C data (Dixon et al., 2012), choosing parameters that maximized overlap with published Hi-C TAD boundaries 
(Methods; Fig. 3a; Supplementary Fig. 5a-d). Of the boundaries identified by GAM, 95\% matched a boundary identified by Hi-C, whilst $76 \%$ of $\mathrm{Hi}-\mathrm{C}$ boundaries matched a boundary in GAM (Fig. 3b), comparable to the overlap of $60 \%$ for boundaries detected from the same Hi-C data using two different computational algorithms (Fig. 3c).

We compared the strength of boundaries detected by only one method with those detected by both GAM and Hi-C. We find that TAD boundaries identified by Hi-C but not GAM (750 boundaries), or by GAM but not Hi-C (123 boundaries), have weaker insulation than common boundaries (Fig. 3d). To investigate whether the boundaries that are differentially detected between Hi-C and GAM have any distinctive features, we plotted the enrichment of various chromatin marks over TAD boundaries. We confirm the enrichment of previously described features at common boundaries (e.g. common boundaries are enriched for CTCF binding, H3K36me3 and SINE elements, but not for H3K9me3; Fig. 3e; Supplementary Fig. 5e). In most cases, features found at common boundaries are also found at boundaries identified only in GAM or only in Hi-C except with lower enrichment, again suggesting that the strongest boundaries are detected by both methods. The exceptions to this are H3K4me3, RefSeq TSSes, unphosphorylated RNA Polymerase II and CTCF, for which there was a small enrichment at Hi-C only boundaries but not at the smaller number of GAM-only boundaries (Supplementary Fig. 5e). TAD boundaries identified by Hi-C but not by GAM may therefore be a consequence of the fact that GAM detects more contacts between TADs and at longer distances, consistent with the lower Hi-C insulation score of these boundaries and the lack of any specific molecular features distinguishing them from common boundaries.
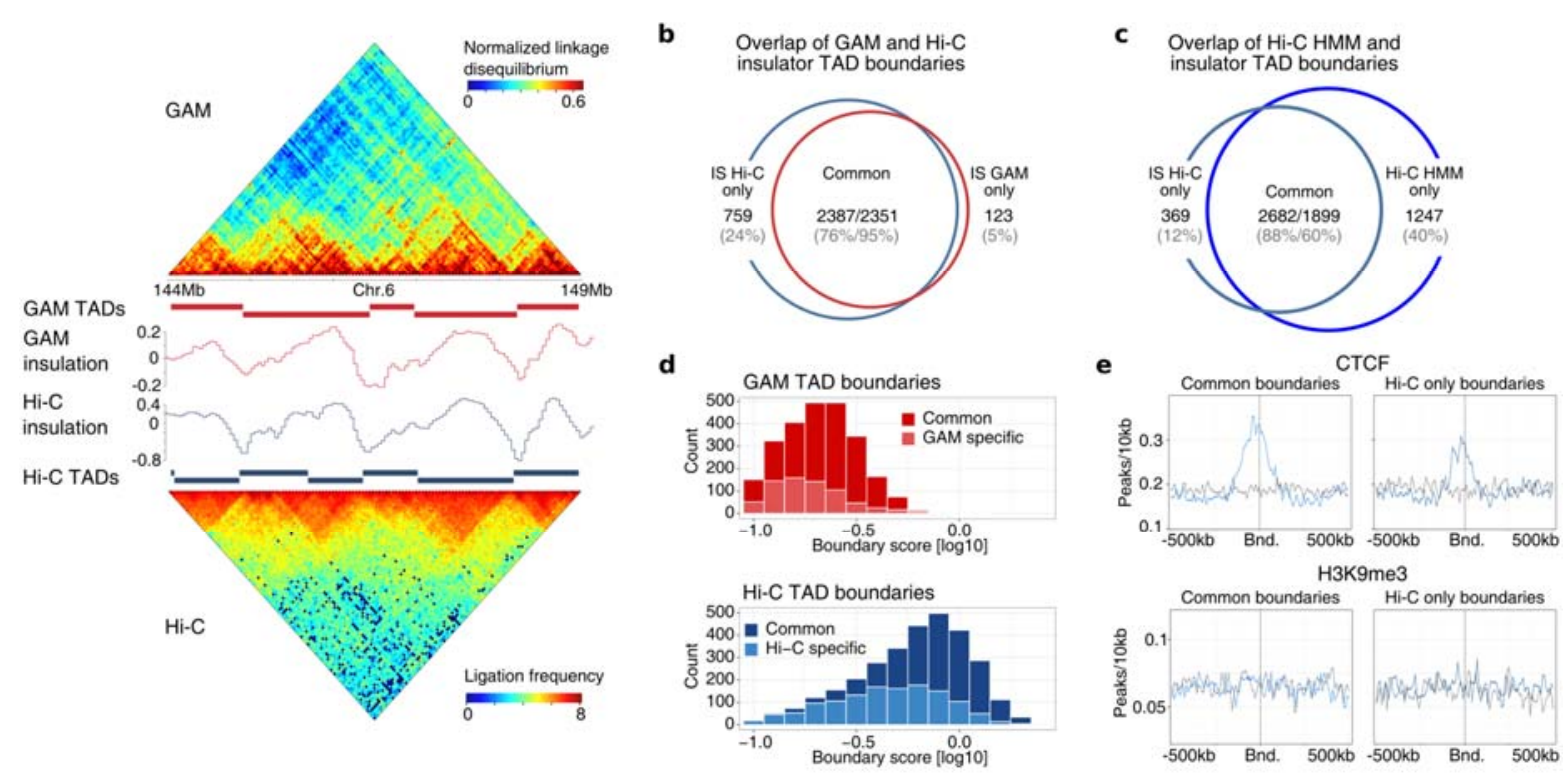

Figure 3

a, Comparison Topological associated domains (TADs) defined by the insulation score method in GAM (top) and Hi-C (Dixon et al., 2012; bottom). b, Overlap (within $80 \mathrm{~kb}$ distance) of TAD boundaries detected in GAM (red) and Hi-C (blue). c, Overlap between insulator score and Hidden Markov Model (HMM) TAD boundary calls from the same Hi-C data. d, Boundary strength (drop of insulation score) measured by GAM (red) and Hi-C (blue) for TAD boundaries that are shared between the two methods (dark bars) or only found in a single method (light bars). e, Enrichment 
(blue) of CTCF and H3K9me3 around common or Hi-C-specific TAD boundaries (Bnd.), compared to genomic background using shuffled boundary positions (grey). Enrichments for GAM-only boundaries are not shown as these are very few.

To investigate genome-wide differences between GAM and Hi-C in an unbiased fashion, we developed a method for directly comparing matrices derived from the two methods. For these analyses, we considered contacts between loci separated by $\leq 4 \mathrm{Mb}$, as the fidelity of both datasets decreases at larger genomic distances, and our simulated multiplex-GAM dataset confirmed that most of the contact information in pairwise matrices is well preserved within this distance when combining NPs in sets of three (Supplementary Fig. 1d). The selected genomic length scale is useful in most current applications of chromatin contact mapping, in particular, it is sufficient for the detection of enhancer-promoter contacts in most instances.

Because GAM detects far more contacts at larger genomic distances (Kempfer and Pombo, 2020) and because data generated by the two techniques has very different numerical distributions, it remained challenging to identify regions with similar or divergent contact patterns between GAM and Hi-C. GAM normalized linkage values of genomic window cosegregation range from -1 to +1 , whereas Hi-C ligation frequency is dependent on sequencing depth and ranges over many orders of magnitude. We therefore first applied a distance-based z-score normalisation to both datasets to address the distance-decay (Fig. 4a). Next, we subtracted the two normalized matrices and extracted the most divergent contacts where the difference between the two matrices was greater than the $5 \%$ extremes defined by a fitted Normal distribution. We refer to these most divergent contacts as GAM-specific or Hi-Cspecific contacts (Fig. 4a).

Next, we established a set of strong-and-common contacts that are well detected in both methods by taking the $10 \%$ strongest contacts which showed a z-score delta of less than 1.0. Many of the strong-and-common contacts are also prominent interactions detected by SLICE analyses of GAM data (Fig. 4b). Strong-and-common contacts and SLICE interactions were more frequently separated by genomic distances below $2 \mathrm{Mb}$ (Supplementary Fig. 6a), whereas GAM-specific and Hi-C-specific contacts were slightly depleted at distances $<1 \mathrm{Mb}$ (Supplementary Fig. 6b). To verify that the GAM-specific and Hi-C-specific contacts are indeed strong contacts in each corresponding method (i.e. they are not artefacts of our normalisation procedure), we compared their raw intensities. These analyses confirmed that our approach successfully identifies contacts with high intensity in GAM but low intensity in Hi-C (or vice-versa; Supplementary Fig. 6c). Importantly, whilst many of the strongest contacts in absolute terms were identified by both methods, more than half of the strongest $20 \%$ of contacts in GAM or Hi-C were method specific (Fig. 4c). 
a

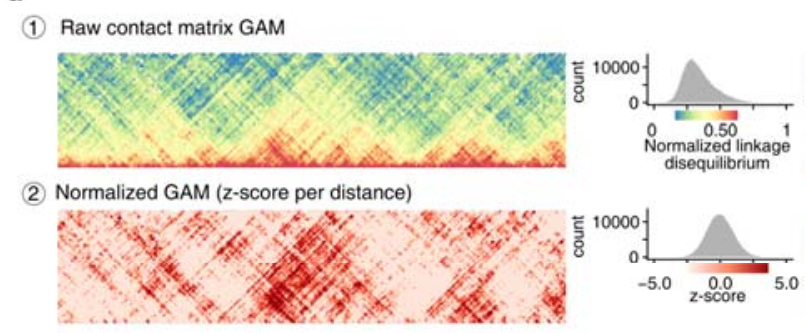

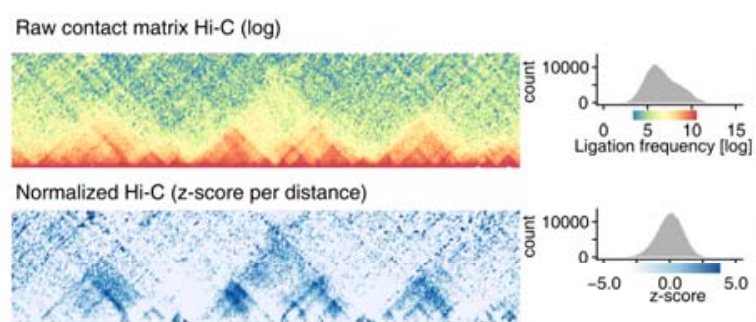

(3) Delta z-scores (GAM - Hi-C)
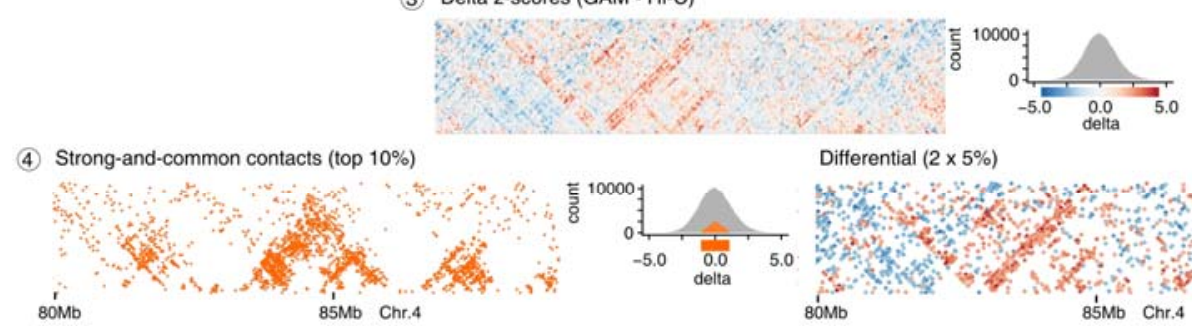

Differential $(2 \times 5 \%)$

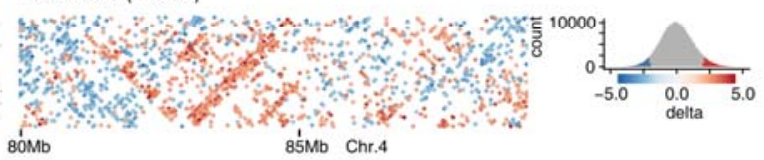

b

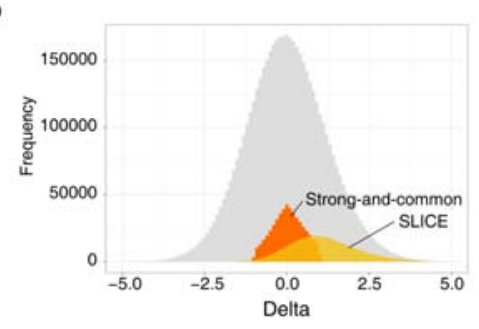

c

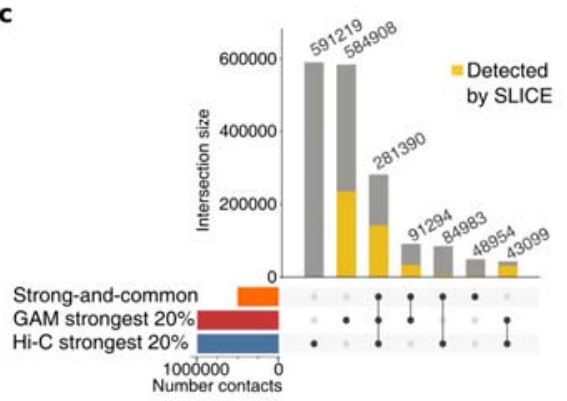

Figure 4

a, Strategy to assess differences and similarities between GAM and Hi-C contact maps. GAM and $\mathrm{Hi}$ $\mathrm{C}$ contact data have very different distributions (1), so contacts at the same genomic distance are normalized by z-score transformation (2). Hi-C z-scores are then subtracted from GAM z-scores to generate a delta $\mathrm{Z}$-score matrix (3), from which we extract the top $10 \%$ of contacts common for both methods (strong-and-common; 4) and the most differential contacts between GAM and Hi-C (GAMspecific or Hi-C specific; 5). b, Prominent interactions identified by SLICE shown within the distribution of delta Z-scores. c, Set sizes for contacts found in combinations of the top $20 \%$ strongest contacts from GAM and Hi-C, and the strong-and-common set. Split bars indicate fraction of each set supported by SLICE prominent interactions.

To investigate whether the contacts differentially detected by GAM or Hi-C hold important biological roles, we asked whether they were enriched for particular genomic features. We created a dataset of features including repeat elements, heterochromatin marks, transcription factor binding sites, RNA Polymerase II and transcription-related histone marks (Fig. 5a). We then counted the number of contacts in each category (GAM-specific, Hi-C-specific, strongand-common) between each possible pair of features (e.g. CTCF-CTCF, EP300-Nanog, etc.), and looked for feature pairs over-represented (enriched) or under-represented (depleted) from GAM-specific or Hi-C-specific contacts relative to distance-matched random backgrounds (Methods; Supplementary Fig. 7a). We found many feature pairs enriched in GAM-specific contacts, whereas only a small number were enriched in Hi-C-specific contacts (Supplementary Fig. 7b,c). The feature pairs most enriched in GAM-specific contacts included transcription factors, enhancers and RNA Polymerase II (Fig. 5b). By contrast, 
heterochromatin regions (i.e. those marked by $\mathrm{H} 3 \mathrm{~K} 9 \mathrm{me} 3$ or $\mathrm{H} 3 \mathrm{~K} 20 \mathrm{me} 3$ ) were most enriched in Hi-C-specific contacts.

As this analysis is conducted at $40 \mathrm{~kb}$ resolution, many contact anchors may contain multiple genomic features. We therefore stratified our analysis to account for overlapping feature pair annotations (Fig. 5c). For example, the GAM-specific, Hi-C-specific and strong-and-common contact sets all contain large numbers of CTCF-CTCF contacts, being enriched over the genome-wide background in the strong-and-common set and GAM-specific contacts, but depleted in Hi-C-specific contacts. Amongst the GAM-specific contacts, the most common subgroup of CTCF-CTCF contacts consisted of those in which contact anchors also overlapped enhancers and the elongating form of RNAPII (a marker of active genes; 14,403 of 61,212 total CTCF-CTCF contacts). By contrast, in Hi-C-specific and strong-and-common contacts, the most common CTCF-CTCF contacts were those without any other annotation (Hi-C specific: 5,930 of 25,236 total CTCF-CTCF contacts; strong-and-common: 20,710 of 89,533). These results suggest that GAM-specific contacts are strongly enriched for a specific subset of CTCF-CTCF contacts that co-occur with enhancer and active genes and which are underestimated in Hi-C data. Indeed, the enhancer-CTCF class of contacts was the most informative in a random-forest model trained to distinguish GAM-specific and Hi-C specific contacts (Fig. 5d). 

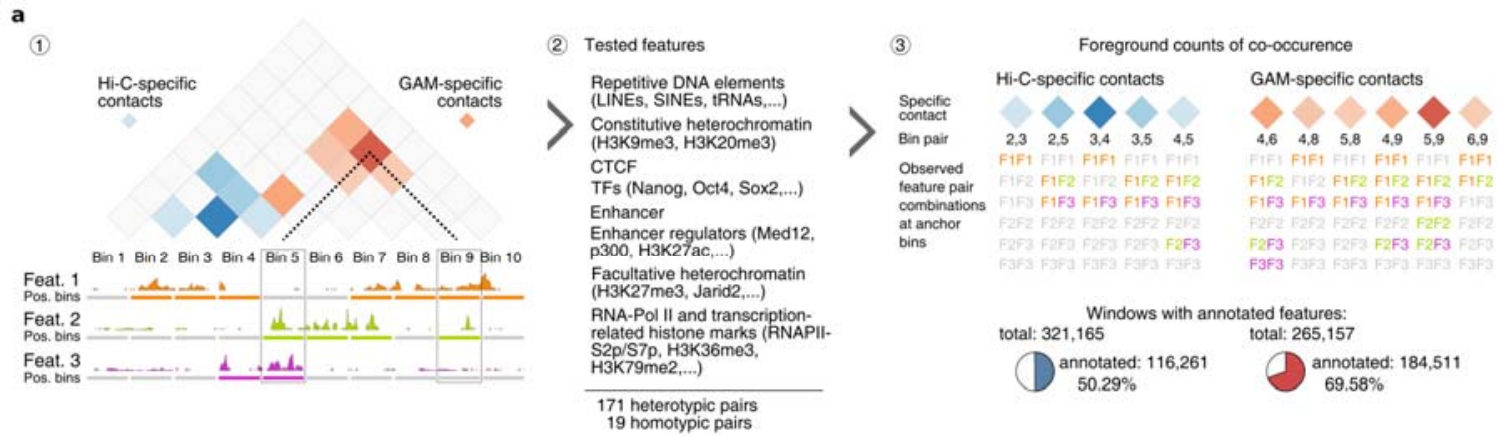

b
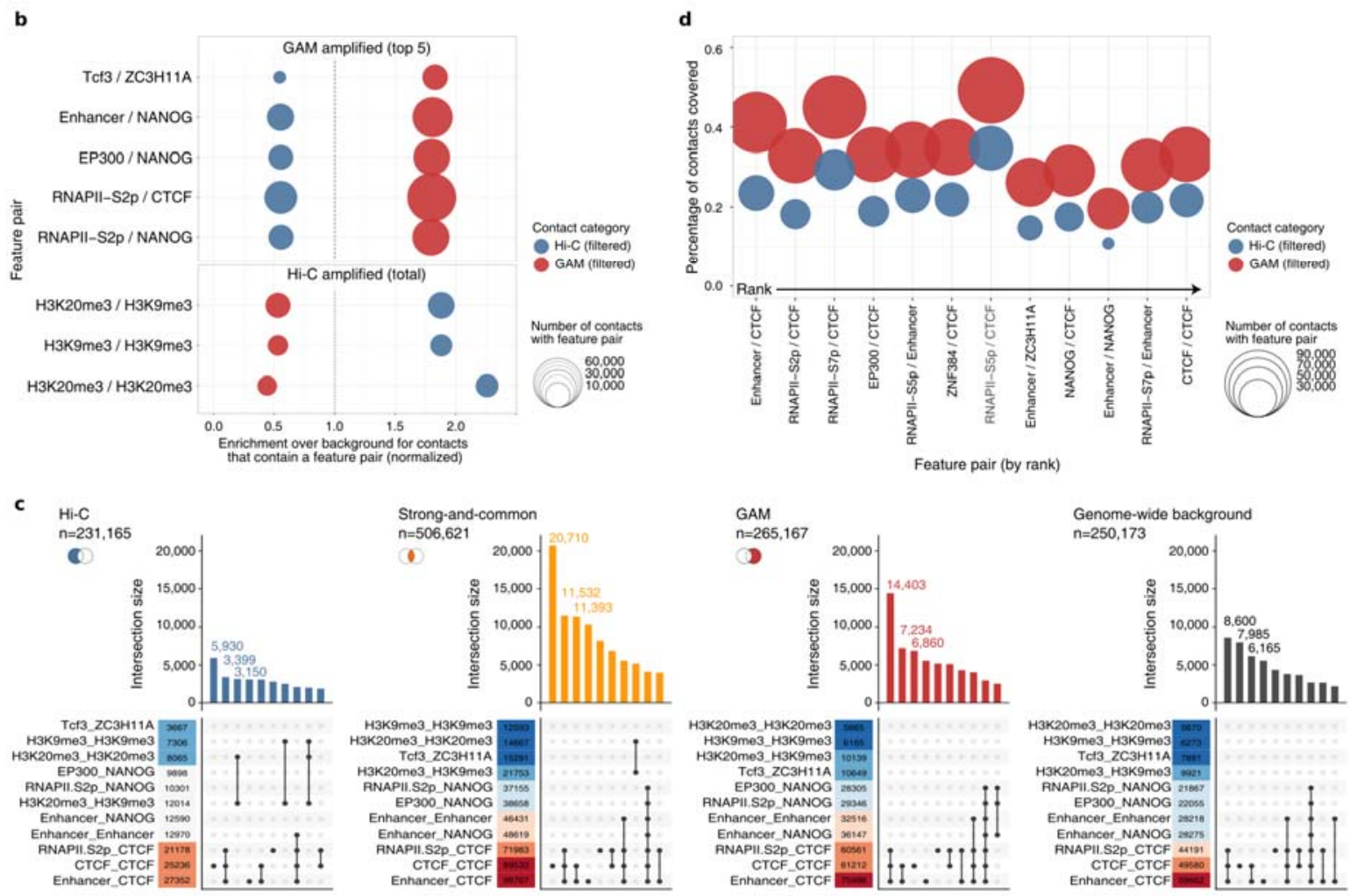

Figure 5

a, Schematic for detecting feature pair enrichments in method-specific contacts (red and blue). (1) Each contact is defined by two genomic anchor points which we categorized as either positive or negative for peaks of the respective feature. (2) We assessed 190 feature pairs and (3) quantified feature occurrences at the anchor points, filtering out contacts with no feature pairs. b. Feature pairs with the highest enrichment over background for GAM-specific (top) and Hi-C-specific (bottom) contacts. c, Upset plots quantifying the co-occurrences of enriched feature pairs in contacts of each subgroup. Occurrence counts for each feature pair are coloured according to their absolute frequency within contacts of each set. d, Feature pairs with the highest discriminatory power (by mean decrease of Gini impurity) in random forests trained to separate GAM-specific and Hi-C-specific contacts.

Having identified striking enrichments for specific genomic features amongst GAM- and Hi-C-specific contacts, we wondered whether specific features might be generally poorly detected in contacts specific to either method. To identify blind-spots in each method, we developed an approach that counted the number of GAM-specific and Hi-C-specific contacts 
formed by each window and looked for regions involved in many more GAM-specific than Hi-C-specific contacts or vice versa (Fig. 6a; Supplementary Fig. 8a). Surprisingly, we find that blind-spot windows are not only abundant but also cluster in the linear genome, such that large regions consistently interact more strongly either in GAM or in Hi-C (Fig. 6b). Clusters that form many GAM-specific contacts (GAM-preferred regions) contained more genes and had higher transcriptional activity (Fig. 6c) whilst clusters that form many Hi-C-specific contacts (Hi-C preferred regions) were more frequently found in regions associated with the nuclear lamina (Fig. 6d). We further identified that GAM-preferred regions tend to be occupied by CTCF, P300, certain mES-cell TFs, RNA Polymerase II (especially the elongating, S2p form), enhancers and super-enhancers, whereas Hi-C-preferred regions showed a slight enrichment for the heterochromatin marks H3K20me3 or H3K9me3 (Fig. 6e; Supplementary Fig. 8b). 
b
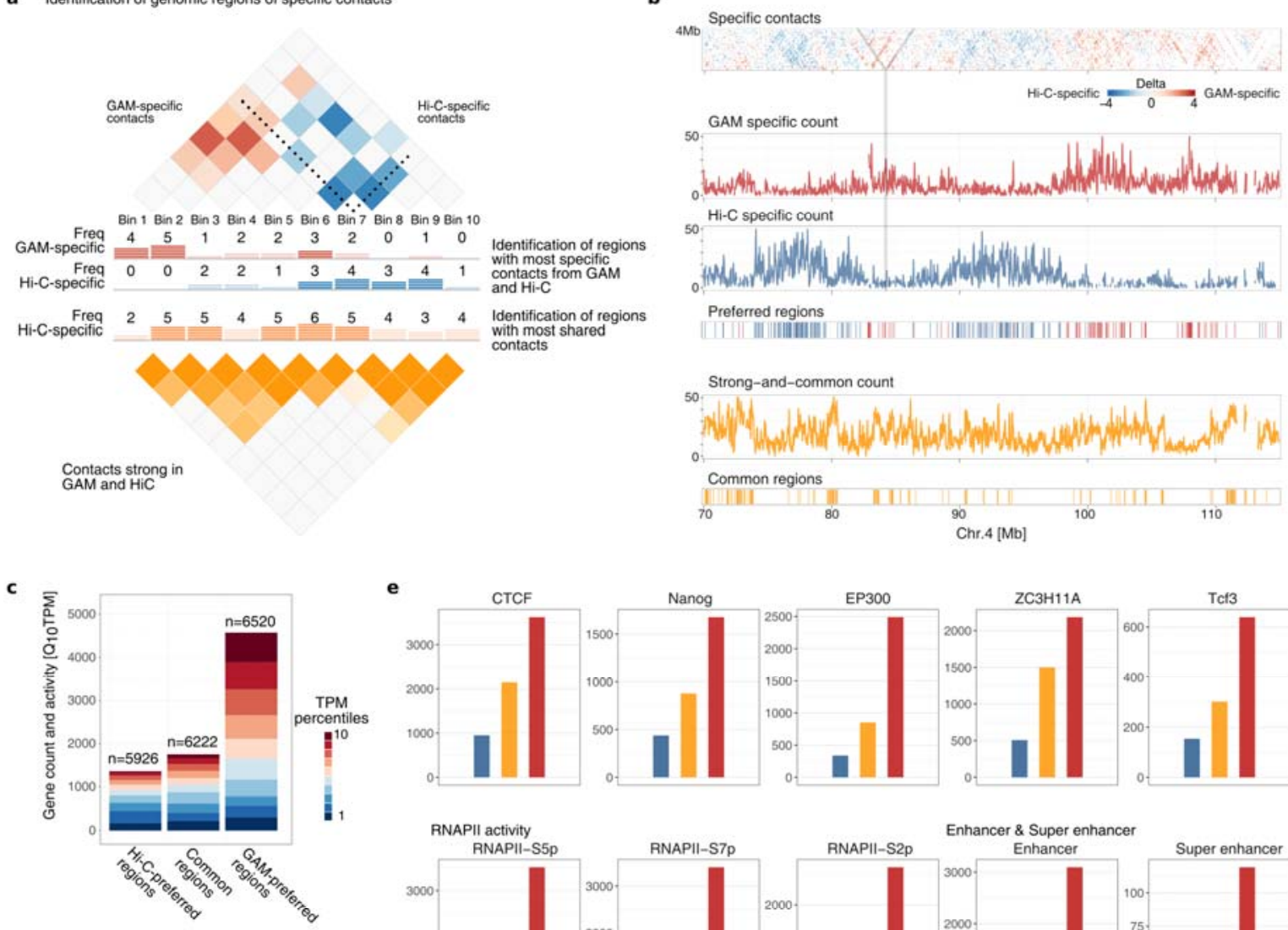

e
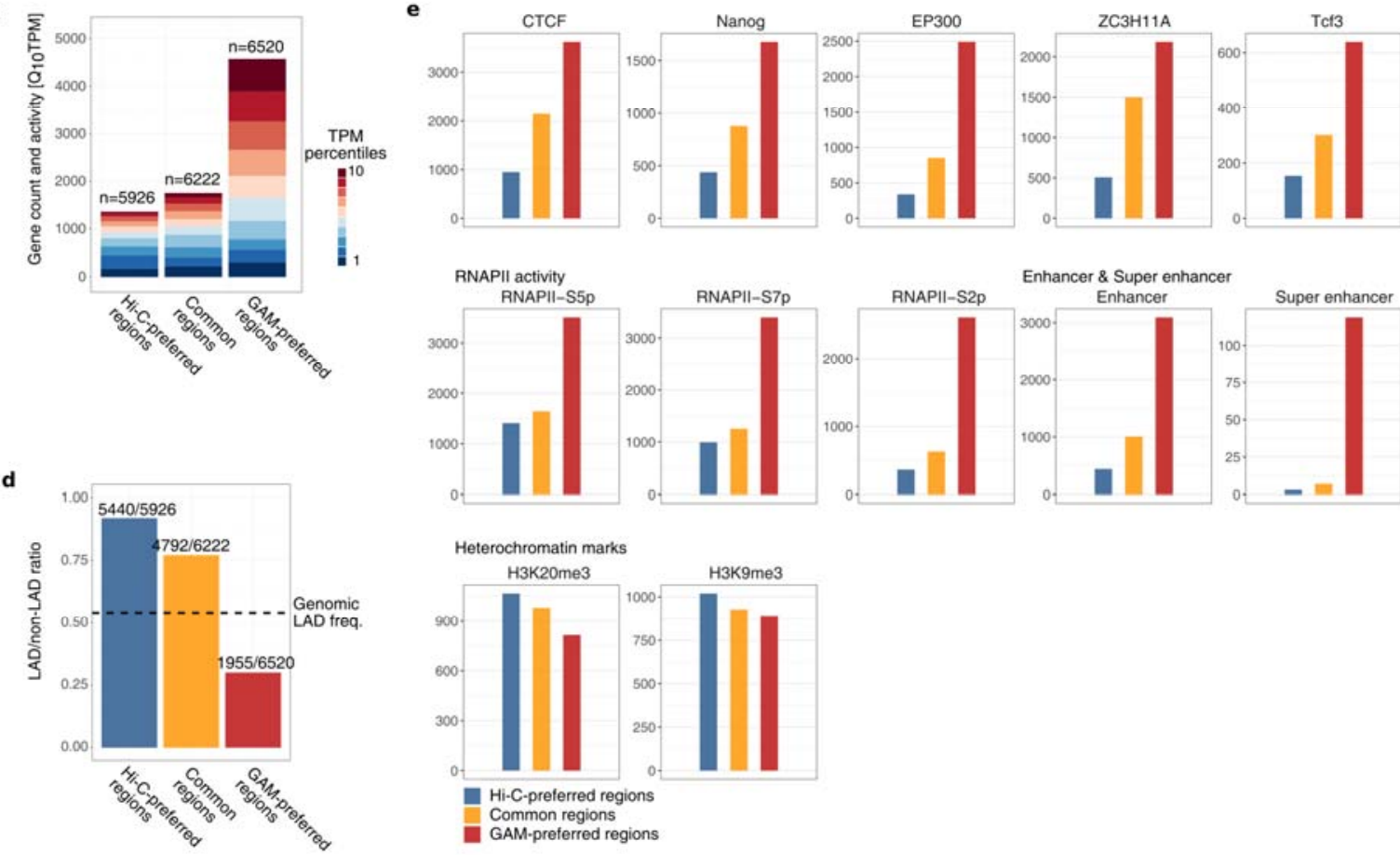

\section{Figure 6}

a, Strategy for identifying genomic regions forming many contacts specific to either GAM or Hi-C. We counted how often a genomic region was found to be an anchor point in the set of GAM-specific contacts, Hi-C-specific contacts, or strong-and-common contacts. The $10 \%$ of genomic windows with the highest absolute difference between the number of GAM-specific and Hi-C-specific contacts were classified as Hi-C-preferred regions or GAM-preferred regions, respectively. Similarly, top $10 \%$ of strong-and-common contacts were used to define common regions seen equally well by both methods. b. Example region on chromosome 4 showing delta z-score matrix (top) and clusters of methodspecific preferences designated as Hi-C-preferred regions, GAM-preferred regions or common regions (bottom). c, Number of genes and distribution of gene transcriptional activity (TPM) for HiC-preferred (left), common (middle) and GAM-preferred (right) regions. d, Fraction of genomic windows within Lamina Associated Domains (LADs) for Hi-C-preferred (left), common (middle) and GAM-preferred (right) regions. Numbers on top of the bar give absolute numbers for LAD windows over the total of windows per category. e, Numbers of genomic windows overlapping a range of features. 
Since the observed patterns can be partly explained by compartmentalisation, we wondered why GAM and Hi-C might measure different relative contact strengths for these compartments. First, we computed compartment definitions from GAM and Hi-C data. Next, we measured the proportion of GAM- or Hi-C-preferred regions that belong to compartments A or B (the active or inactive compartments, respectively), and found that GAM-preferred regions are highly enriched for chromatin belonging to compartment A, whereas Hi-Cpreferred regions and strong-and-common regions are enriched within the B compartment (Fig. 7a; Supplementary Fig. 9).

Finally, we considered whether the enrichment in GAM-preferred regions for active features (active genes, TFs, polymerase, enhancers and compartment A), could be due to different levels of contact complexity, i.e. to interactions with many simultaneous interacting partners (Fig. 7b). Complex interactions are expected to be underestimated in $\mathrm{Hi}-\mathrm{C}$ datasets because the ligation step only allows for the measurement of two interacting partners per restriction fragment in each cell where the contact is established (O'Sullivan et al., 2013).

To investigate the relationship between interaction complexity and the differences in methodspecific contacts and method-preferred regions, we used SLICE to predict the probability of interaction for all possible sets of three $1 \mathrm{Mb}$ windows lying on the same chromosome (i.e. the $P i_{A B C}$ for all possible triplets of loci A, B and C; Beagrie et al., 2017). We then calculated the mean $P i_{A B C}$ across all triplets involving a particular window. Mean $P i_{A B C}$ measures the number of distinct interactions formed by each window that have at least two other partners, and is therefore a measure of interaction complexity. We find that windows in the A compartment indeed form more complex interactions than windows in the B compartment (Fig. 7c). Interestingly, windows that formed many GAM-specific contacts had a much higher complexity than windows that establish strong-and-common or Hi-C-specific contacts even when comparing within the same compartment. All the genomic features associated with GAM-specific contacts were also associated with greater interaction complexity, with the strongest effect seen for the elongating, S2-phosphoisoform of RNA Polymerase II and for super-enhancers (Fig. 7d), in line with our previous work which identified long-range chromatin contacts between super-enhancers and actively-transcribed genomic regions across tens of megabases (Beagrie et al., 2017). These results suggest the existence of abundant chromatin contacts where many active regions interact simultaneously, which have been so far unappreciated through the use of ligation-based methods, but detected by GAM and FISH (Beagrie et al., 2017).

Finally, we examined whether high interaction complexity might artificially deflate pair-wise contact probability as measured by Hi-C, since each DNA fragment picks up a given interacting fragment with lower probability in complex compared with simple contacts (Fig. $7 b)$. We correlated pairwise contacts from GAM and $\mathrm{Hi}-\mathrm{C}$ at $1 \mathrm{Mb}$ resolution and found that regions with a high complexity generally have higher pair-wise contact scores when measured by GAM, whereas regions of low complexity generally have higher pair-wise 
contact scores when measured by Hi-C (Fig. 7e). This is particularly evident when plotting only a subset of interacting pairs that all have very similar GAM contact frequency (Fig. 7e, zoomed inset), demonstrating that complexity explains a large amount of the divergence in contact frequencies measured by $\mathrm{Hi}-\mathrm{C}$ or GAM.

a

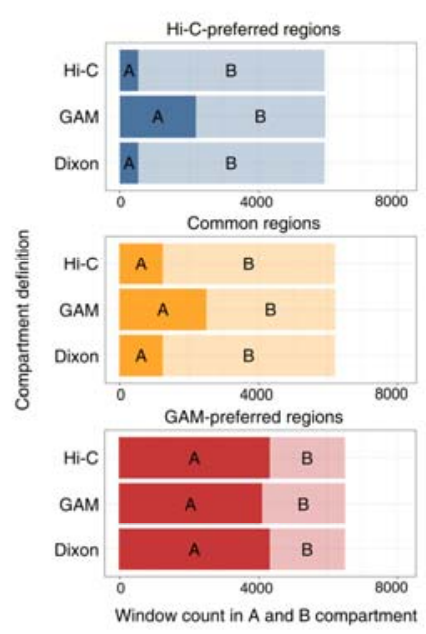

d

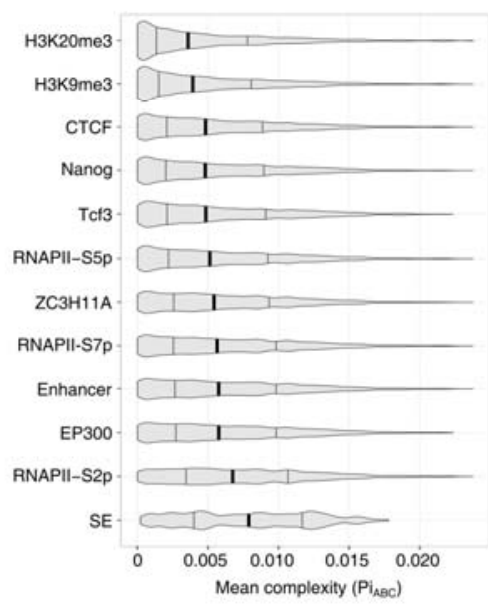

b
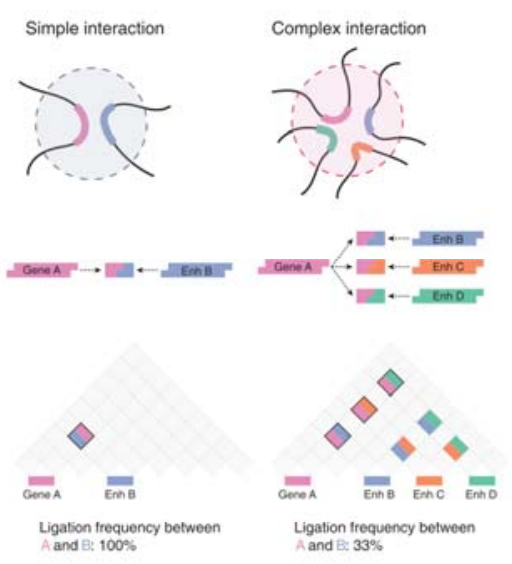

e

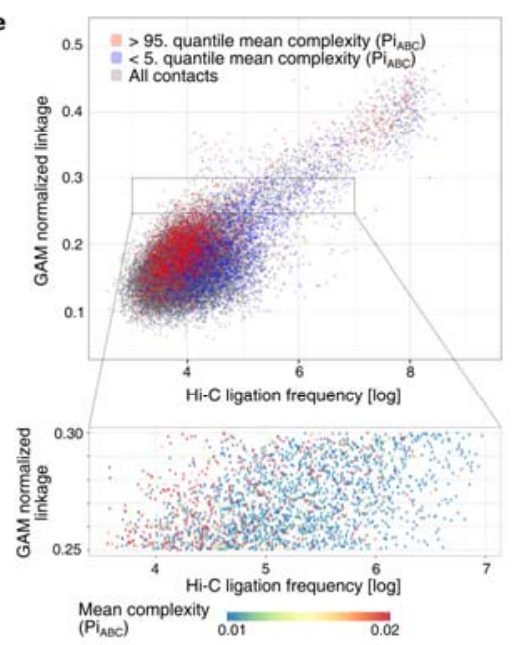

c
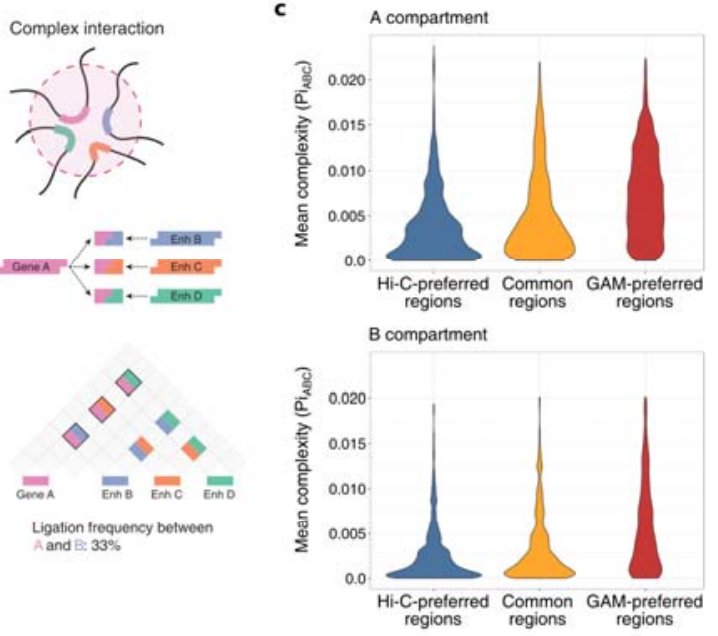

Figure 7

a, Number of windows designated as A or B compartments by PCA decomposition of Hi-C data or GAM data, or from published Hi-C PCA compartments (Dixon et al., 2012) in Hi-C-preferred (top), common (middle) and GAM-preferred (bottom) regions. b, Interaction complexity: simple interactions involve only a few genomic regions, whilst complex interactions involve many genomic regions at once. Ligation events connect two pieces of DNA, so pairwise contacts can be directly determined while measurement of higher order contacts may be affected by competing ligation events. c, Contact complexity (measured as the mean $\mathrm{Pi}_{\mathrm{ABC}}-$ the interaction probability of $1 \mathrm{Mb}$ triplets calculated by SLICE) for Hi-C-preferred (left), common (middle) or GAM-preferred (right) regions in the A compartment (top) or the B compartment (bottom). d, Complexity for genomic windows which are positive for a feature, ranked by increasing mean. Vertical bars mark the $25 \%$, $50 \%$ and $75 \%$ quantiles. e, Relationship between pairwise Hi-C ligation frequency (X-axis), pairwise GAM normalized linkage (Y-axis) and complexity (5\% pairwise interactions with highest mean $P i_{A B C}$ in red, lowest $5 \%$ in blue). Inset: Hi-C ligation frequency of $1 \mathrm{Mb}$ pairwise contacts that have a similar GAM normalized linkage, coloured by complexity. 


\section{Discussion}

The three-dimensional structure of the nucleus is inextricably linked with its functional roles, including gene regulation, DNA replication and the DNA damage response. Consequently, molecular techniques for measuring the $3 \mathrm{D}$ folding of chromatin within the nucleus have been instrumental in advancing our understanding of nuclear function over the past decade (Kempfer and Pombo, 2020). Here, we have developed multiplex-GAM, a new variant of Genome Architecture Mapping that enables faster and more cost-effective analysis of chromatin folding genome-wide than the previous version (Beagrie et al., 2017). GAM requires only a few hundred cells, which is of particular relevance to human genetics where researchers need to assay the $3 \mathrm{D}$ contacts made by disease-linked sequence variants in specific, often rare cell types impacted by the disease (e.g. neuronal subtypes in neurodegenerative diseases). We have recently applied Multiplex-GAM to different neuronal subtypes in brain tissues, and discovered abundant cell-type specific contacts that contain differentially expressed genes and accessible regulatory elements (Winick-Ng et al., 2020).

Here, we develop multiplex-GAM to generate a larger GAM dataset, and expand the mathematical model SLICE by incorporating new experimental parameters (number of NPs per sample and nuclear ellipticity). We use the larger GAM dataset containing information from 1250 cells for detailed comparisons of the contacts captured by GAM and Hi-C, the most frequently used genome-wide method for chromatin contact analysis (Lieberman-Aiden et al., 2009). We find that GAM and Hi-C detect similar TADs, large folded domains that are thought to constrain gene regulatory elements and form a fundamental unit of chromatin organisation (Dixon et al., 2012; Nora et al., 2012; Sexton et al., 2012). Many strong contacts, including a large proportion of CTCF-mediated loops, are also detected by both methods. By careful examination of finer-scale differences, we identify that chromatin contacts given more weight by GAM frequently connect genomic loci bound by enhancers, key mES-cell transcription factors, RNA Polymerase II and CTCF, whereas contacts which feature more prominently in Hi-C matrices connect regions marked by the heterochromatinassociated histone modifications $\mathrm{H} 3 \mathrm{~K} 9 \mathrm{me} 3$ and $\mathrm{H} 3 \mathrm{~K} 20 \mathrm{me} 3$.

We then looked for regions of the genome which consistently form more contacts in GAM datasets than in $\mathrm{Hi}-\mathrm{C}$ datasets and found that these regions are located in large genomic regions bound by the same activating transcription factors identified in the GAM-specific contacts. In our previous work, we found that super-enhancers are the genomic regions most enriched in complex, multi-partner interactions, together with the most actively transcribed regions (Beagrie et al., 2017). We now extend this finding to show that the regions forming stronger contacts in GAM than in $\mathrm{Hi}-\mathrm{C}$ tend to form more complex interactions. Theoretical work has previously suggested that ligation-based methods, such as Hi-C, might underestimate contacts between multiple partners, as ligation only captures two or a few contact partners at a time (O'Sullivan et al., 2013). We find that ligation frequencies 
measured by Hi-C are systematically lower between regions that form complex interactions, providing the first experimental evidence to support such an effect.

Ligation is not the only potential source of difference between the two methods, as GAM and $\mathrm{Hi}-\mathrm{C}$ also make use of quite different fixation protocols. The choice of fixation protocol has been shown to affect the proportion of informative ligation events between different $3 \mathrm{C}$ experiments (Oudelaar et al., 2017), and it may also influence the contacts of genomic regions with different protein composition and/or compaction within a single experiment (Downes et al., 2021; Williamson et al., 2014). The digestion of nuclear chromatin necessary for preparing Hi-C libraries has also been shown to disrupt nuclear structure (Gavrilov et al., 2013), whereas GAM uses fixation protocols specifically chosen to maximize the preservation of nuclear architecture and retention of nuclear proteins (Guillot et al., 2004). Ultimately, formaldehyde fixation remains a "black box" and will continue to complicate interpretation of the most widely used methods for measuring chromatin structure (including microscopy methods like FISH; Gavrilov et al., 2015). Live-cell imaging methods circumvent the need for fixation and will provide valuable orthogonal data, but these methods currently require recruitment of large numbers of fluorophores which may themselves influence folding (Shaban and Seeber, 2020). Ultimately, it should eventually be possible to shed light on the effect of fixation by extending GAM to unfixed nuclei through sectioning of vitrified samples.

Another factor that may influence method-specific contacts is data processing. It has recently been shown that $\mathrm{Hi}-\mathrm{C}$ detects fewer contacts between regions of condensed chromatin due to a lower accessibility of these regions to restriction enzyme digestion (Chandradoss et al., 2020). However, matrix-balancing algorithms commonly used to normalize Hi-C data can overcorrect for this effect, leading to an aberrantly high frequency of contacts between condensed domains. Consistent with these results, we find that regions of the genome which consistently form more contacts in normalized Hi-C are enriched for heterochromatin marks. We have found the bias in raw GAM datasets to be uniformly lower than that found in raw $\mathrm{Hi}-\mathrm{C}$, yet both methods have their own specific biases and improved normalisation algorithms have the potential to bridge the divergences between the two methods (Beagrie et al., 2017; Chandradoss et al., 2020; Kruse et al., 2020; Liu and Wang, 2019).

Our work underscores previous findings that complex, simultaneous interactions between many partners are a pervasive and under-studied feature of mammalian genome architecture (Beagrie et al., 2017; Quinodoz et al., 2018), although their overall prevalence is still a subject of debate (Olivares-Chauvet et al., 2016). Enhancer-binding transcription factors and RNA Polymerase II have both been reported to form nuclear clusters that could serve as nucleating agents for such multi-partner interactions (Iborra et al., 1996; Liu et al., 2014). More recently, there has been a surge of interest in phase-separated nuclear bodies, which are suggested to facilitate high local concentrations of chromatin-interacting proteins and/or transcriptional regulators (Yoshizawa et al., 2020). The clear expectation is that these condensates should bring together multiple interacting genomic partners, in much the same way as rDNA repeats are brought together in the nucleolus (Pederson, 2011). 
Heterochromatin has also been reported to form phase-separated condensates (Larson et al., 2017), yet we find these regions to have lower-complexity specific interactions, potentially highlighting a shorter-range role for these interactions.

In conclusion, our development of multiplex-GAM, an improved protocol for rapid, costeffective generation of GAM datasets enabled us to obtain a deeper GAM dataset for mES cells and to explore the similarities and differences between GAM and Hi-C in greater detail. Reassuringly, the two methods paint broadly similar pictures of nuclear architecture, in particular the distribution of TADs, the segregation of nuclear chromatin into A and B compartments and the importance of CTCF for shaping chromatin interactions. There are differences however, with GAM detecting more and stronger contacts between active chromatin regions, and across longer distances, and Hi-C emphasising contacts within silent chromatin. These results highlight the utility of GAM for studying contacts of potential generegulatory functions, particularly in human disease where such contacts may only be formed in rare cell populations inaccessible to population $\mathrm{Hi}-\mathrm{C}$.

\section{Methods}

\section{Updated GAM protocol}

mES cells were grown and cryoblocks prepared as previously described (Beagrie et al., 2017). $220 \mathrm{~nm}$ (green) cryosections were cut with glass knives using a Leica FC7 ultracut cryotome, collected in sucrose droplets $(2.1 \mathrm{M}$ in PBS) and transferred to steel frame PEN membrane slides (Leica) UV treated for 45 min prior to use. Slides were washed in sterilefiltered $(0.2 \mu \mathrm{m}$ syringe filter) $1 \mathrm{x}$ PBS ( 3 times, $5 \mathrm{~min}$ each), then with sterile-filtered water (3 times, $5 \mathrm{~min}$ each). Cresyl violet staining was performed with sterile-filtered cresyl violet ( $1 \% \mathrm{w} / \mathrm{v}$ in water, Sigma-Aldrich, C5042) for $10 \mathrm{~min}$, followed by 2 washes with water (30 s each) and air dryed for $15 \mathrm{~min}$. Nuclear profiles were laser microdissected into adhesive 8strip LCM collection caps (Zeiss), with four profiles dissected into each cap. Caps were stored at $-20{ }^{\circ} \mathrm{C}$ until ready to proceed with whole genome amplification.

Whole genome amplification of DNA from microdissected NPs was performed using the Sigma WGA4 kit using a liquid handling robot (Microlab STARlet, Hamilton). $14.5 \mu \mathrm{l}$ of lysis and fragmentation mastermix $\left(13 \mu \mathrm{H} \mathrm{H}_{2} \mathrm{O}, 1.4 \mu \mathrm{l}\right.$ lysis and fragmentation buffer, $0.09 \mu \mathrm{l}$ proteinase K) was added to each well of a 96 well plate, caps with microdissected material were used to close the wells, then the plate was inverted and centrifuged upside-down at 3000 $\mathrm{xg}$ for 2 min such that the fragmentation mastermix was collected in the cap. Plates were incubated upside-down for $4 \mathrm{~h}$ at $50{ }^{\circ} \mathrm{C}$ then inverted and centrifuged right-way-up at 3000 $\mathrm{xg}$ for 2 min to collect the extracted DNA in the bottom of the well. Samples were then heat inactivated at $99{ }^{\circ} \mathrm{C}$ for 4 min then cooled on ice for 2 min. $4.95 \mu$ library preparation mastermix (3.3 $\mu$ l library preparation buffer, $1.65 \mu$ library stabilisation solution) was added 
to each sample, incubated at $95{ }^{\circ} \mathrm{C}$ for $2 \mathrm{~min}$, cooled on ice for $2 \mathrm{~min}$ then centrifuged at $3000 \mathrm{xg}$ for $2 \mathrm{~min}$. $4.5 \mu \mathrm{l}$ library preparation enzyme (diluted $3 \mathrm{x}$ with $\mathrm{H}_{2} \mathrm{O}$ ) was added to each tube, then samples were incubated at $16{ }^{\circ} \mathrm{C}$ for $20 \mathrm{~min}$, then $24{ }^{\circ} \mathrm{C}$ for $20 \mathrm{~min}, 37^{\circ} \mathrm{C}$ for $20 \mathrm{~min}$ and $75^{\circ} \mathrm{C}$ for $5 \mathrm{~min}$. Finally, $85 \mu \mathrm{l}$ amplification master mix (11 $\mu \mathrm{l}$ amplification buffer, $66.5 \mu \mathrm{l} \mathrm{H}_{2} \mathrm{O}, 7.5 \mu \mathrm{l}$ WGA polymerase) was added to each tube and samples were amplified by PCR (initial denaturation $95{ }^{\circ} \mathrm{C}$ for $3 \mathrm{~min}$, then 24 cycles of denaturation $95{ }^{\circ} \mathrm{C}$ for $30 \mathrm{~s}$ and annealing/extension $65^{\circ} \mathrm{C}$ for $5 \mathrm{~min}$ ).

Amplified DNA was purified using Ampure XP beads. $61.5 \mu \mathrm{l}$ beads were mixed with $77 \mu \mathrm{l}$ amplified sample in a fresh 96-well plate and incubated at room temperature for $5 \mathrm{~min}$. The plate was placed on a magnetic stand for $5 \mathrm{~min}$, then the supernatant was discarded and the beads were washed twice with $200 \mu$ freshly prepared $80 \%$ ethanol. After the second ethanol wash was discarded, the beads were air dried for 5 min and then resuspended in $45 \mu 1 \mathrm{H}_{2} \mathrm{O}$ and incubated at room temperature for $5 \mathrm{~min}$. The plate was then placed on a magnetic stand and the supernatant transferred to a fresh 96-well plate ready for NGS library prep.

Libraries were prepared using the Illumina Nextera library preparation kit following the manufacturer's instructions. DNA concentration of the final libraries was determined using a Picogreen fluorescence assay (ThermoFisher) and libraries were pooled at equimolar concentration ready for sequencing on an Illumina NextSeq machine.

\section{Data processing}

Multiplex-GAM sequencing reads were aligned to the mouse mm9 genome assembly using bowtie2, then positive windows were called by GAMtools v0.0.1 using a fixed read threshold of 4 . Normalized linkage disequilibrium (D') matrices at $40 \mathrm{~kb}$ genomic resolution were generated by GAMtools (Beagrie and Schueler, 2017).

\section{SLICE analysis}

To convert pair or triplet co-segregation frequencies to interaction probabilities (Pi), we computed the segregation probabilities $v_{i}$ for a single locus under assumption of spherical shape, with average nuclear radius $\mathrm{R}$ (estimated by use of cryosection images as equal to $4.5 \mu \mathrm{m}$; Beagrie et al., 2017). The co-segregation probabilities $u_{i}$ for pairs of loci in a notinteracting state have been estimated from GAM segregation data; for interacting loci, instead, we estimated co-segregation $t_{i}$ probabilities by assuming their physical distance less than slice thickness $(h \simeq 220 \mathrm{~nm})$. From linear combinations of these probabilities, by use of "mean-field" approximation, we computed the probability of locus segregation in a nuclear profile for pairs $\left(N_{i, j}\right)$ and triplets $\left(N_{i, j, k}\right.$; Supplementary Note).

The expected number of nuclear profiles $M_{i}$ with 0,1 or 2 loci, are therefore computed from $N_{i, j}$ probabilities. From these, in turn, it is also possible estimate the co-segregation ratio $M_{l} /\left(M_{1}+M_{2}\right)$, i.e., the fraction of tubes with two loci on all non-empty tubes. Since the 
equations describing the tubes content depend on the interaction probability $P i$, the latter can be estimated by fitting the experimental value of co-segregation ratio (Supplementary Note). The same procedure has been used to estimate the probability of triplet interaction. Significant SLICE contacts are those with a co-segregation ratio greater than the $95^{\text {th }}$ percentile of the expected distribution of co-segregation ratios for two non-interacting loci at the given genomic distance.

To apply SLICE to merged multiplex-GAM dataset, we used a mean field approximation. It consists of introducing a non-integer number of nuclear profiles per tube, obtained as the average of different number of nuclear profiles NPs in the different datasets, weighted with corresponding number of tubes (Supplementary Note).

\section{Creation of in silico merged multiplex-GAM data}

Segregation tables (in which each row corresponds to a genomic window, each column to a GAM library and the entries indicate the presence or absence of each window in each NP) were generated from 1NP GAM libraries. A new segregation table was then generated by randomly selecting two, three or four columns from the original table (i.e. $2 / 3 / 4 \mathrm{x} 1 \mathrm{NP}$ libraries), combining them into a single column such that the new column is positive if any of the original columns were positive, and removing the columns from the original table. This procedure was performed iteratively until all columns from the original table had been combined. The new, in silico combined table was then used for the calculation of normalized linkage disequilibrium matrices.

\section{SLICE enrichment tests}

Enrichment of Active/Enhancer/Inactive/Intergenic windows in pairwise SLICE interactions and analysis of triplet SLICE interactions was carried out as previously described (Beagrie et al., 2017).

\section{TAD calling}

For calling TADs, we aimed to exclude potential effects of using different TAD callers for GAM and Hi-C. Thus, we applied the insulation square method (Crane et al., 2015) to GAM matrices of normalized linkage disequilibrium scores and to $\mathrm{Hi}-\mathrm{C}$ matrices of normalized ligation frequencies (GSE35156; Dixon et al., 2012), both at $40 \mathrm{~kb}$ resolution. We adjusted the insulation square method to also consider negative values from GAM normalized linkage disequilibrium and applied it to contact matrices of each chromosome (im mean, ids 50000, nt 0.1 , yb 1.5, bmoe 3). While TAD sizes were nearly insensitive to the size of the insulation square for Hi-C data (reaching plateau around $500 \mathrm{~kb}$ square size; Supplementary Figure 5a), increased sizes of the insulation square produced larger TADs for GAM data. Here, we selected a window size of $400 \mathrm{~kb}$ for GAM and $\mathrm{Hi}-\mathrm{C}$ data which maximizes the agreement between both TAD sets as well as to the HMM TAD boundaries published for the Hi-C dataset (Dixon et al., 2012). To consider differences in the number of detected TAD 
boundaries, we assessed the agreement between two TAD sets by calculating the product of the distances to the respective closest boundary between both sets with either reference.

We used bedtools v2.27.1 (Quinlan AR and Hall IM, 2010) to check if obtained TAD boundaries were touching or overlapping and merged border ranges keeping their maximum boundary score. In comparisons between TAD boundaries from different datasets we considered boundaries to be matching when the distance reported by bedtools closest was at maximum 1 bin.

We checked for abundance of features at the TAD boundaries centred at their boundary midpoints. For a given genomic mark, we analyzed the mean signal within $500 \mathrm{~kb}$ around the identified boundary midpoint in windows of $10 \mathrm{~kb}$ resolution using bedtools. We estimated the background by randomizing the boundary positions using chromosome-wise circular shifts.

\section{Generating peak and feature data}

We mapped genomic and epigenomic read data to the NCBI Build 37/mm9 reference genome using Bowtie2 v2.1.0 (Langmead et al., 2009). We excluded replicated reads (i.e., identical reads, mapped to the same genomic location) found more often than the 95th percentile of the frequency distribution of each dataset. We obtained peaks using BCP v1.1 (Chen et al, 2012) in transcription factor mode or histone modification mode with default settings. A full list of all features analyzed in this study is given in Supplementary Table 2. We computed mean counts of features for all genomic $40 \mathrm{~kb}$ windows by the bedtools window and intersect functions.

\section{PCA compartments}

We computed compartments on GAM and Hi-C data as described (Beagrie et al., 2017; Lieberman-Aiden et al., 2009) or used published compartments definitions (Dixon et al., 2012).

\section{Identification of differential contacts}

GAM and Hi-C implement two different approaches to assess chromatin structure and measure underlying contact frequencies. Comparing the value distributions from GAM D' and log-scaled Hi-C ligation frequencies highlights differences in the distributions that need to be considered. To directly compare contact intensities between both methods genome-wide in an unbiased fashion, we devised a strategy where we first normalize contact frequency matrices, subtract normalized contact intensities, and, ultimately, define strong contacts seen in both or at significantly different levels by either of the two methods.

In order to avoid amplification of spurious contacts from noise and zero inflation, we limited our analysis to $4 \mathrm{Mb}$ genomic distance. From GAM contact data at $40 \mathrm{~kb}$ resolution, we 
removed all contacts with negative D' values. We also excluded all contacts established between potentially oversampled or undersampled genomic windows. Here, we used the relative number of slices with a positive window (window detection frequency, $w d f$ ) as a proxy for detectability and removed windows with a $w d f$ of less than $5 \%$ or above $20 \%$. From the Hi-C data, we excluded all contacts for which zero ligations events were detected. All contacts excluded from any of both datasets were not considered in the definition of differential contacts.

For each chromosome, we applied the z-score transformation to GAM and Hi-C contacts of given distance $\mathrm{d}$. We found this transformation to yield a stable transformation for value intensities from GAM and Hi-C contact matrices (Supplementary Fig. 6c), each resulting in a distribution with a very good fit to a Normal distribution that accounts for the observed decay of mean contact frequency over distance.

To identify the differential contacts between GAM and Hi-C, we computed a delta matrix for each chromosome by subtracting the normalized Hi-C matrix from the normalized GAM matrix. Next, we selected the most differential contacts based on the expected value intensities by fitting a Normal distribution to the distribution of delta values of each chromosome (fitdistrplus R package). We defined Hi-C-specific and GAM-specific contacts to be located within the expected $5 \%$ and $95 \%$ tails of the fitted distributions, which resulted in sets of 231,165 and 265,157 contacts, respectively. Similarly, we defined a set of strongand-common contacts which have little difference in the normalized value intensities from GAM and Hi-C. We ranked all contacts with a delta z-score of less than 1.0 according to the lower $\mathrm{z}$-score value from GAM and $\mathrm{Hi}-\mathrm{C}$, and extracted the strongest $10 \%$ of contacts for each chromosome (total 506,621 contacts).

\section{Feature enrichments within differential contacts}

We queried whether contacts identified to be specific for GAM and $\mathrm{Hi}-\mathrm{C}$ are associated with specific biological features. First, we checked for the occurrence of homotypic and heterotypic feature combinations in the subsets of Hi-C-specific, GAM-specific, strong-andcommon contacts. We annotated $50.29 \%, 69.58 \%$, and $60.28 \%$ of contacts with feature pairs, respectively (Supplementary Fig. 7a).

To assess the amplification of genomic feature pairs in GAM-specific, Hi-C-specific, and strong-and-common contacts, we produced three permutations of random background sets for each of the foreground sets. Here, we randomly sampled the same number of contacts out of all contacts of the same chromosome with the same genomic distance. To determine whether feature combinations are observed in the foreground sets more frequently than to be expected, we computed the amplification (relative frequency) in contrast to the matched background.

Pairs of different features can often be found co-present at the same anchor points of a contact. We applied the UpSetR package (Conway et al., 2017) to the sets of contacts from 
GAM-specific, Hi-C-specific, strong-and-common, and the genome-wide background and plotted the abundance of a feature pair according to the percentile of feature occurrence along with the number of observed colocalization events between pairs of features. We established the genomic background set by randomly selecting $5 \%$ of all non-zero contacts obserbed by GAM and Hi-C.

To directly compare GAM-specific and Hi-C-specific contacts and to see whether both sets can be distinguished using the associated feature pairs, we trained a Random Forest model (sklearn 0.19.2, 100 trees, no pruning) and extracted the top 12 feature pairs that were most informative for binary classification based on the Gini importance criterion.

\section{Analysis of GAM-preferred and Hi-C-preferred regions}

We assessed for each genomic $40 \mathrm{~kb}$ window the preference towards preferentially producing GAM-specific contacts or Hi-C-specific contacts. First, we counted how often a window was an anchor point for contacts of the GAM-specific or Hi-C-specific subsets. Next, we calculated the absolute difference between both counts and estimated a $90 \%$ percentile cutoff for each chromosome. We considered genomic windows above this threshold to either hold mostly GAM-specific contacts or mostly Hi-C-specific contacts. In total, this resulted in 6,520 windows to be labelled as GAM-preferred regions by having predominantly GAMspecific contacts, and 5,926 Hi-C-preferred regions with a much higher count of Hi-Cspecific contacts over GAM-specific contacts. Similarly, we identified genomic regions that are equally well detected by GAM and Hi-C. Here, we counted for each genomic window the number of anchor points from contacts of the strong-and-common set and selected the top $10 \%$ genomic windows with the highest counts from each chromosome.

Next, we assessed gene density and transcriptional activity in groups of genomic regions using published gene annotations and mESC-46C TPM values (Ferrai et al., 2017). We transferred provided mm10 gene positions to mm9 using UCSC liftover (Kuhn et al., 2013) and assigned genes to genomic windows of $40 \mathrm{~kb}$ using bedtools intersect. We annotated lamina associations within $40 \mathrm{~kb}$ genomic regions according to $\mathrm{mESC}$ LaminB1 HMM calls (Peric-Hupkes et al., 2010). The genome-wide LAD ratio was computed by the number of positive HMM state calls over the total number of windows. For markers of TFs, histone modifications, and RNA Polymerase II states, we used $40 \mathrm{~kb}$ window classification for peak and feature presence (Supplementary Table 2) and counted the number of positive windows in each subset.

\section{Analysis of interaction complexity}

We used SLICE to compute the three-way probability of interaction $\left(P i_{A B C}\right)$ for all potential $1 \mathrm{Mb}$ intrachromosomal triplets from the GAM-1250 dataset (Supplementary Note). We define complexity as the mean $P i_{A B C}$ - either the mean over all combinations of "B" and "C" 
windows for a given "A" window (where complexity is being calculated for a genomic region, Fig. 7c,d) or the mean over all "C" windows for a given "A" and "B" window (where complexity is calculated for a pairwise contact, Fig. 7e).

For each genomic window labelled as Hi-C preferred regions, common regions, or GAMpreferred regions, we checked for the compartment assignment and correlated the outcome with the complexity at the $1 \mathrm{Mb}$ genomic window. Similarly, we estimated the complexity of genomic and epigenetic features by subsetting $40 \mathrm{~kb}$ genomic windows according to the presence of TFs, histone modifications, and RNA polymerase II states and presenting the complexity of the respective $1 \mathrm{Mb}$ genomic window.

\section{Acknowledgments}

The authors thank all lab members and the 4D nucleome consortium for helpful discussions. We thank Warren Winick-Ng for providing illustrations in Fig. 1c,d. We also deeply appreciate the feedback from Teresa Szczepińska on the processing of differential contacts.

AP acknowledges support from the Helmholtz Association (Germany). AP and MN acknowledge support from the National Institutes of Health Common Fund 4D Nucleome Program grant U54DK107977, and the Berlin Institute of Health (BIH). AP acknowledges support from the Deutsche Forschungsgemeinschaft (DFG; German Research Foundation) under Germany's Excellence Strategy - EXC-2049 - 390688087. MN thanks for support from CINECA ISCRA Grant HP10CYFPS5 and HP10CRTY8P, by computer resources at INFN and Scope at the University of Naples (MN). LW acknowledges the support of Ohio University's GERB program. RAB is supported by the Wellcome Trust (209181/Z/17/Z).

\section{Data availability}

The original GAM sequencing data (Beagrie et al., 2017) are available from GEO (GSE64881). GAM sequencing data generated for this study are available as a separate accession (GSE166381).

\section{Code availability}

GAM sequencing samples were processed using GAMtools v1.0.0 which is available at https://github.com/pombo-lab/gamtools/releases/tag/v1.0.0. Custom Python code used for data analysis will be made available at https://github.com/pombo-lab/multiplex-gam-2021.

\section{Author contributions}

$\mathrm{AP}$ and $\mathrm{MN}$ devised the multiplex-GAM strategy;

$\mathrm{RAB}, \mathrm{AK}$ and RK produced GAM datasets;

$\mathrm{RAB}, \mathrm{AK}$ and RK optimised the experimental protocol;

RAB performed GAM data processing, QC and in-silico merging experiments;

CA, AS, SB, AMC, and MN developed and implemented the updated SLICE model;

CA and AS applied SLICE to find optimal experimental parameters and performed SLICE analysis of GAM data;

RAB performed enrichment analyses for SLICE contact pairs and triplets; 
CJT and MS performed TAD boundary analysis;

CJT and AK processed epigenetic and genomic data;

CJT and MS devised pipeline to extract differential contacts;

CJT generated randomized contact sets;

YZ and YL developed and applied the pipeline for feature pair presence and enrichments

over background;

CB performed Random Forest discrimination tests;

$\mathrm{CB}$ and CJT performed feature pair co-localization tests;

CJT identified and analysed preferred regions;

RAB and CJT assessed complexity of contacts, preferred regions, and feature-positive genomic windows;

AP supervised GAM experiments and bioinformatics analyses;

LW supervised feature enrichment analyses in specific contacts;

MN supervised SLICE development;

AP, RAB, CJT, AK, CA, AS, YZ, CB, LW, and MN contributed to the interpretation of the results;

RAB wrote the first draft of the manuscript;

$\mathrm{RAB}$ and CJT designed the figures;

$\mathrm{RAB}, \mathrm{CJT}$, and AP wrote the manuscript;

All authors provided critical feedback and helped revise the manuscript.

The authors consider RAB, CJT and CA to have contributed equally to this work.

\section{Competing interests}

AP, MN, RAB and AS hold a patent on 'Genome Architecture Mapping': Pombo, A., Edwards, P. A. W., Nicodemi, M., Beagrie, R. A. \& Scialdone, A. Patent

PCT/EP2015/079413 (2015).

\section{Materials \& Correspondence}

Correspondence and material requests should be addressed to ana.pombo@mdc-berlin.de; mario.nicodemi@na.infn.it; welch@ohio.edu

\section{References}

Andersson, R., and Sandelin, A. (2020). Determinants of enhancer and promoter activities of regulatory elements. Nat. Rev. Genet. 21, 71-87.

Beagan, J.A., and Phillips-Cremins, J.E. (2020). On the existence and functionality of topologically associating domains. Nat. Genet. 52, 8-16.

Beagrie, R.A., and Schueler, M. (2017). GAMtools: an automated pipeline for analysis of Genome Architecture Mapping data. BioRxiv 10.1101/114710.

Beagrie, R.A., Scialdone, A., Schueler, M., Kraemer, D.C.A., Chotalia, M., Xie, S.Q., Barbieri, M., de Santiago, I., Lavitas, L., Branco, M.R., et al. (2017). Complex multienhancer contacts captured by genome architecture mapping. Nature 543, 519-524.

Bonev, B., Cohen, N.M., Szabo, Q., Hugnot, J., Tanay, A., Cavalli, G., Bonev, B., Cohen, N.M., Szabo, Q., Fritsch, L., et al. (2017). Multiscale 3D Genome Rewiring during Mouse 
Article Multiscale 3D Genome Rewiring during Mouse Neural Development. Cell 171, 557.e1-557.e24.

Chandradoss, K.R., Guthikonda, P.K., Kethavath, S., Dass, M., Singh, H., Nayak, R., Kurukuti, S., and Sandhu, K.S. (2020). Biased visibility in Hi-C datasets marks dynamically regulated condensed and decondensed chromatin states genome-wide. BMC Genomics 21, 175.

Conway, J.R., Lex, A., and Gehlenborg, N. (2017). UpSetR: an R package for the visualization of intersecting sets and their properties. Bioinformatics 33, 2938-2940.

Crane, E., Bian, Q., McCord, R.P., Lajoie, B.R., Wheeler, B.S., Ralston, E.J., Uzawa, S., Dekker, J., and Meyer, B.J. (2015). Condensin-driven remodelling of X chromosome topology during dosage compensation. Nature 523, 240-244.

Dixon, J.R., Selvaraj, S., Yue, F., Kim, A., Li, Y., Shen, Y., Hu, M., Liu, J.S., and Ren, B. (2012). Topological domains in mammalian genomes identified by analysis of chromatin interactions. Nature 485, 376-380.

Downes, D.J., Beagrie, R.A., Gosden, M.E., Telenius, J., Carpenter, S.J., Nussbaum, L., De Ornellas, S., Sergeant, M., Eijsbouts, C.Q., Schwessinger, R., et al. (2021). Highresolution targeted $3 \mathrm{C}$ interrogation of cis-regulatory element organization at genome-wide scale. Nat. Commun. 12, 531.

Ferrai, C., Torlai Triglia, E., Risner $\square$ Janiczek, J.R., Rito, T., Rackham, O.J., Santiago, I., Kukalev, A., Nicodemi, M., Akalin, A., Li, M., et al. (2017). RNA polymerase II primes Polycomb $\square$ repressed developmental genes throughout terminal neuronal differentiation. Mol. Syst. Biol. 13, 946.

Gavrilov, A., Razin, S. V, and Cavalli, G. (2015). In vivo formaldehyde cross-linking: it is time for black box analysis. Brief. Funct. Genomics 14, 163-165.

Gavrilov, A. a, Gushchanskaya, E.S., Strelkova, O., Zhironkina, O., Kireev, I.I., Iarovaia, O. V, and Razin, S. V (2013). Disclosure of a structural milieu for the proximity ligation reveals the elusive nature of an active chromatin hub. Nucleic Acids Res. 41, 3563-3575.

Guillot, P. V, Xie, S.Q., Hollinshead, M., and Pombo, A. (2004). Fixation-induced redistribution of hyperphosphorylated RNA polymerase II in the nucleus of human cells. Exp. Cell Res. 295, 460-468.

Iborra, F.J., Pombo, A., Jackson, D. a, and Cook, P.R. (1996). Active RNA polymerases are localized within discrete transcription "factories" in human nuclei. J. Cell Sci. 109, 14271436.

Kempfer, R., and Pombo, A. (2020). Methods for mapping 3D chromosome architecture. Nat. Rev. Genet. 21, 207-226.

Kruse, K., Hug, C.B., and Vaquerizas, J.M. (2020). FAN-C: a feature-rich framework for the analysis and visualisation of chromosome conformation capture data. Genome Biol. 21, 303.

Kuhn, R.M., Haussler, D., and Kent, W.J. (2013). The UCSC genome browser and associated tools. Brief. Bioinform. 14, 144-161.

Larson, A.G., Elnatan, D., Keenen, M.M., Trnka, M.J., Johnston, J.B., Burlingame, A.L., Agard, D.A., Redding, S., and Narlikar, G.J. (2017). Liquid droplet formation by HP1 $\alpha$ suggests a role for phase separation in heterochromatin. Nature 547, 236-240.

Lieberman-Aiden, E., van Berkum, N.L., Williams, L., Imakaev, M., Ragoczy, T., Telling, A., Amit, I., Lajoie, B.R., Sabo, P.J., Dorschner, M.O., et al. (2009). Comprehensive mapping of long-range interactions reveals folding principles of the human genome. Science 326, 289-293.

Liu, T., and Wang, Z. (2019). normGAM: an R package to remove systematic biases in 
genome architecture mapping data. BMC Genomics 20, 1006.

Liu, Z., Legant, W.R., Chen, B.-C., Li, L., Grimm, J.B., Lavis, L.D., Betzig, E., and Tjian, R. (2014). 3D imaging of Sox 2 enhancer clusters in embryonic stem cells. Elife 3, e04236.

Nora, E.P., Lajoie, B.R., Schulz, E.G., Giorgetti, L., Okamoto, I., Servant, N., Piolot, T., van Berkum, N.L., Meisig, J., Sedat, J., et al. (2012). Spatial partitioning of the regulatory landscape of the X-inactivation centre. Nature 485, 381-385.

O’Sullivan, J.M., Hendy, M.D., Pichugina, T., Wake, G.C.G., and Langowski, J. (2013). The statistical-mechanics of chromosome conformation capture. Nucleus 4, 390-398.

Olivares-Chauvet, P., Mukamel, Z., Lifshitz, A., Schwartzman, O., Elkayam, N.O., Lubling, Y., Deikus, G., Sebra, R.P., and Tanay, A. (2016). Capturing pairwise and multiway chromosomal conformations using chromosomal walks. Nature 540, 296-300.

Oudelaar, A.M., Davies, J.O.J., Downes, D.J., Higgs, D.R., and Hughes, J.R. (2017). Robust detection of chromosomal interactions from small numbers of cells using low-input Capture-C. Nucleic Acids Res. 45, e184.

Pederson, T. (2011). The Nucleolus. Cold Spring Harb. Perspect. Biol. 3, a000638.

Peric-Hupkes, D., Meuleman, W., Pagie, L., Bruggeman, S.W.M., Solovei, I., Brugman, W., Gräf, S., Flicek, P., Kerkhoven, R.M., van Lohuizen, M., et al. (2010). Molecular Maps of the Reorganization of Genome-Nuclear Lamina Interactions during Differentiation. Mol. Cell 38, 603-613.

Quinodoz, S.A., Ollikainen, N., Tabak, B., Palla, A., Schmidt, J.M., Detmar, E., Lai, M.M., Shishkin, A.A., Bhat, P., Takei, Y., et al. (2018). Higher-Order Inter-chromosomal Hubs Shape 3D Genome Organization in the Nucleus. Cell 174, 744-757.e24.

Sexton, T., Yaffe, E., Kenigsberg, E., Bantignies, F., Leblanc, B., Hoichman, M., Parrinello, H., Tanay, A., and Cavalli, G. (2012). Three-dimensional folding and functional organization principles of the Drosophila genome. Cell 148, 458-472.

Shaban, H.A., and Seeber, A. (2020). Monitoring the spatio-temporal organization and dynamics of the genome. Nucleic Acids Res. 48, 3423-3434.

Williamson, I., Berlivet, S., Eskeland, R., Boyle, S., Illingworth, R.S., Paquette, D., and Bickmore, W.A. (2014). Spatial genome organization $\square$ : contrasting views from chromosome conformation capture and fluorescence in situ hybridization. Genes Dev. 28, 2778-2791.

Winick-Ng, W., Kukalev, A., Harabulā, I., Zea Redondo, L., Meijer, M., Serebreni, L., Bianco, S., Szabo, D., Chiariello, A.M., Irastorza Azcarate, I., et al. (2020). Cell-type specialization in the brain is encoded by specific long-range chromatin topologies. BioRxiv 10.1101/2020.04.02.020990.

Yoshizawa, T., Nozawa, R.-S., Jia, T.Z., Saio, T., and Mori, E. (2020). Biological phase separation: cell biology meets biophysics. Biophys. Rev. 12, 519-539. 